\title{
Handover Performance in Mobile WIMAX Networks
}

\author{
Zobida Abbass $^{1}$, Dr. Mohmmed Abakar ${ }^{2}$ \\ Department of Communication Engineering, Al-Neelain University
}

\begin{abstract}
WIMAX is Wireless Interoperability for Microwave Access. It is a telecommunication technology that provides wireless data over long distances in several ways, from point-to-point links to full mobile cellular type access. The main consideration of Mobile WiMAX is to achieve seamless handover such that there is no loss of data. In WiMAX both mobile station (MS) and base station (BS) scans the neighboring base stations for selecting the best base station for a potential handover. Two types of handovers in WiMAX are: Hard handover (break before make) and Soft handover (make before break). To avoid data loss during handover we have considered soft handovers this research topic. We have proposed a technique to select a base station for potential soft handover in WiMAX. We have developed a base station selection procedure that will optimize the soft handover such that there is no data loss; handover decision is taken quickly and thus improving overall handover performance. We will compare the quality of service with hard handover and soft handover. We have analyzed the proposed technique with an existing scheme for soft handover in WiMAX with simulation results.
\end{abstract}

Keywords: WiMAX, Handover, BER, FBSS, MDHO

\section{Introduction}

IEEE 802.16 standard defines the air interface for fixed change in serving base station but it does not necessarily Broadband Wireless Access (BWA) systems to be used in mean that the base station must be changed. WMANs (Wireless Metropolitan Area Networks), commonly referred to as WiMAX (Worldwide Interoperability for Microwave Access).

\section{Types of Handover}

The original There are two types of handovers used in cellular network standard IEEE 802.16 does not support mobility and for systems: hard handover and soft handover. The hard this purpose IEEE 802.16e-2005 was introduced [1]. The handover is used when the communication channel is also known as Mobile WiMAX. When a new mobile is released first a new channel is acquired later from the older version of the older WiMAX specification known as IEEE the neighboring cell. For real-time users it means a short 802.16e-2004 which is wireless but fixed which helps to lack the disconnection of communication. For real-time users it means a short $802.16 \mathrm{e}-2004$ which is wireless but fixed, it lacks the disconnection of communication. Thus, there is service ability for user to move during data transmission. The interruption when the handover occurs reducing the main purpose of WiMAX is to provide users in rural areas quality of service. Hard handover is used by the systems with high speed communications as an alternative to which use time division multiple access (TDMA) and expensive wired connections (e.g. cable or DSL) [2]. That frequency division multiple access (FDMA) such as GSM is WiMAX is capable to provide high speed internet to last and General Packet Radio Service (GPRS) [4]. mile connections. But this is not the only purpose of WiMAX systems. Mobile WiMAX allows the user to A. HARD HANDOVER move freely during data transmission.
The main consideration of mobile WiMAX is that there should be no The entire process of HHO in IEEE 802.16e is broadly data loss when the moving user switches from one base handover. Handover is (NTAP) and the Actuaj Handover phase (AHoP).

\subsection{Handovers in WiMax}

The ability to make a decision is a special requirement of a mobile device; to change its serving base station if there exists another base station with better signal strength in the reach of mobile station (MS). Handover is a procedure that provides continuous connection when a MS migrates from the air interface of one BS to another air-interface provided by another BS without disturbing the existing connections..

Handovers are needed to support mobility. the SBS periodically broadcasts information about the state of the NBSs, preparing for potential handover For a handover to occur, one needs to have at least two activities. The SBS keeps on gathering these channel base stations: serving base station (SBS) and target base information of the NBSs with the help of the backbone station (TBS).Scanning of advertised neighboring BSs by MS: The MS scans the advertised BSs within specific timeframes, to select suitable candidate BSs for the handover. A list of potential candidate TBSs is thus maintained.

This procedure is carried out with the help of Scanning Interval Allocation request and response messages (MOB SCN-REQ and MOB SCN-RSP), respectively, sent by the MS and the SBS. In the end, Scanning Result Report (MOB SCN-REP) summarizes all the scanning activities.

Ranging and Optional Association Activities: The scanning is followed by contention/non-contention ranging activities through which the MS gathers further information about the PHY channel related to the selected TBSs. Ranging Request (RNG REQ) and Ranging 


\section{International Journal of Science and Research (IJSR) \\ ISSN (Online): 2319-7064}

Index Copernicus Value (2013): 6.14 | Impact Factor (2015): 6.391

Response (RNG RSP) messages are used for this purpose. Ranging may be followed by optional association activities through which the MS gets associated with the potential target BS candidates. Association Result Reports (MOB ASC-REP) are used for this purpose.

\subsection{Soft Handover}

The soft handover, in contrast to hard handover, establishes multiple connections with neighboring cells. Soft handover is used by the code division multiple access (CDMA) systems where the cells use same frequency band using different code words. Each MS maintains an active set where BSs are added when the RSS exceeds a given threshold and removed when RSS drops below another threshold value for a given amount of time specified by a timer. When a presence or absence of a BS to the active set is encountered soft handover occurs. The systems using soft handoff are Interim Standard 95 (IS95) and Wideband CDMA (WCDMA).

\subsection{Fast Base Station Switching}

We are considering fast base station switching technique. In this method a diversity set is maintained for each mobile station. The serving base station and mobile station monitors the neighboring base stations that can be added in diversity set. Diversity set is maintained by both mobile station and serving base station. Diversity set is collection of base stations that can be chosen as target base station for a handover.

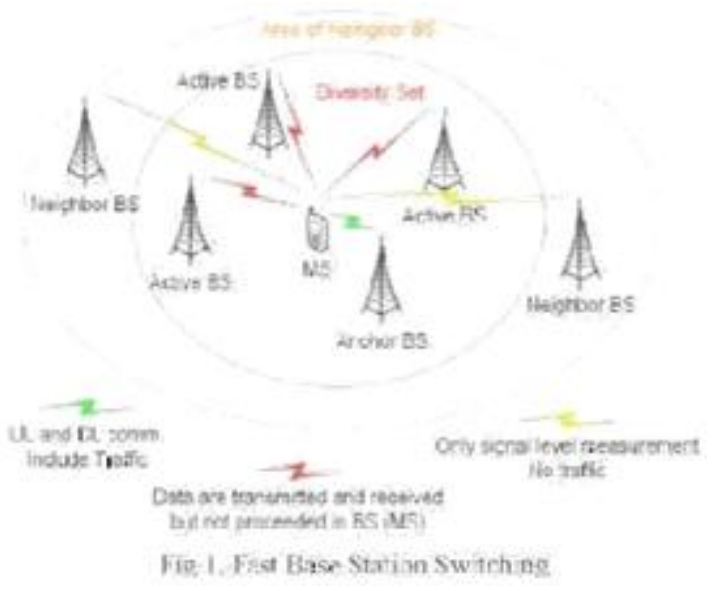

In fig. 1, the mobile station selects one base station from diversity set as anchor base station sends its current location to it which is sent to base station controller for decision of a handover. Whenever there is a need of handover base station controller sends handover initiation message to mobile station. Handover decision can be taken by mobile station, base station or base station controller depending upon the implementation [6].

\subsection{Macro Diversity Handover}

MDHO is designed to allow full seamless mobility at much higher speeds (up to $120 \mathrm{kmph}$ ). It has the advantage of performing handovers within sectors having the same carrier frequency due to their employing the universal reuse concept.

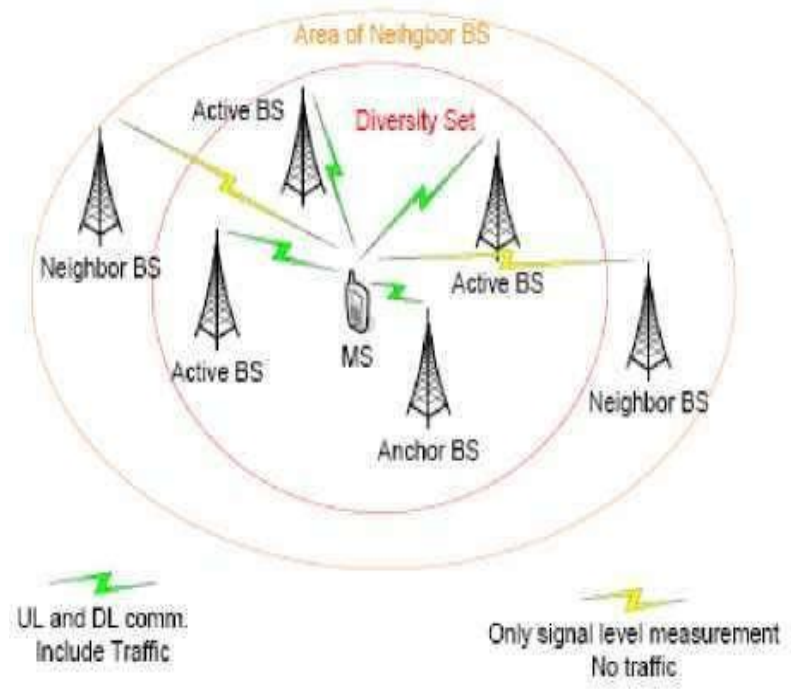

Fig 2. Micro Diversity Handover

Figure 2: Encapsulates the following:

- Diversity set: Diversity set is the group of BS's selected by an MS such that all these BS's operate on same frequency channel and are synchronized on time and frame level. Diversity set is formed by sharing of MAC context between BS and MSS. Also MSS select only those BS's as the member of diversity set whose CINR (Carrier to interference noise ratio).

- Updating the Diversity set: Diversity set may be updated under two situations: first when the CISR level of the serving BS falls below a predefined level known as H_Delete threshold, while the second case any BS is added into the diversity set if it has CISR levels above H_Add threshold.

- Active BS: All the BS's of the diversity set having all the information about the MSS including the MAC context.

- Serving BS: This is the BS to which the MS is recently handed over or registered with.

- Neighbour BS: This is the BS which is not the part of diversity set i.e., no traffic is exchanged with this BS but still MS can perform the signal strength measurement with this BS.

\begin{tabular}{|c|c|c|c|}
\hline \multicolumn{4}{|c|}{ Comparison between soft and hard handover } \\
\hline Parameter & Hard Handover & FBSS & MDHO \\
\hline Latency & High & Medium & Low \\
\hline Complexity & Low & Medium & High \\
\hline Reliability & Low & Medium & High \\
\hline Packet Loss & High & Low & Low \\
\hline Cost & Low & Medium & High \\
\hline Link Quality & Low & Medium & High \\
\hline Speed & Low & Medium & High \\
\hline
\end{tabular}




\section{International Journal of Science and Research (IJSR) \\ ISSN (Online): 2319-7064}

Index Copernicus Value (2013): 6.14 | Impact Factor (2015): 6.391

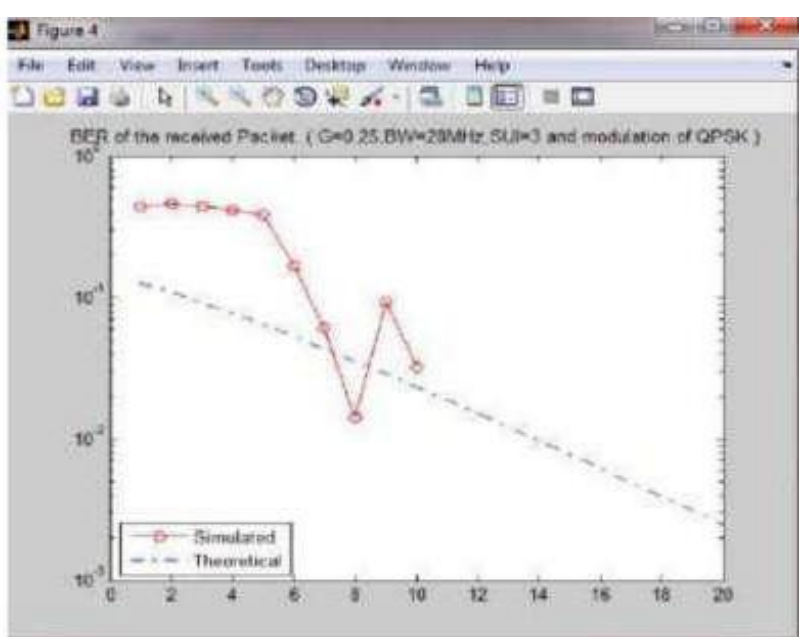

Figure 4: Handover Performance for QPSK channel (BER)

\section{MATLAB Simulink Results}

Handover performance of mobile WiMAX is evaluated by measuring bit error rate versus time lapsed when packets transmitted from source to destination station through different modulation techniques like BPSK, QPSK \& 16QAM. During performance evaluation different parameters like cyclic prefix, no. of OFDM symbols, bandwidth and no. of channels are considered which are entered by user. Following are the steps followed for simulation-

1) Simulations are as (BPSK, QPSK, 16 QAM).

2) Type of "cyclic prefix" (1/4 1/8 1/16 1/32).

3) The simulation WITH and WITHOUT encoding of the bits and study.

4) We carry out the simulation through different SUI channels $(=1)$.

5) We do a simulation with different values of the nominal BW of the system.

6) To exit the program.

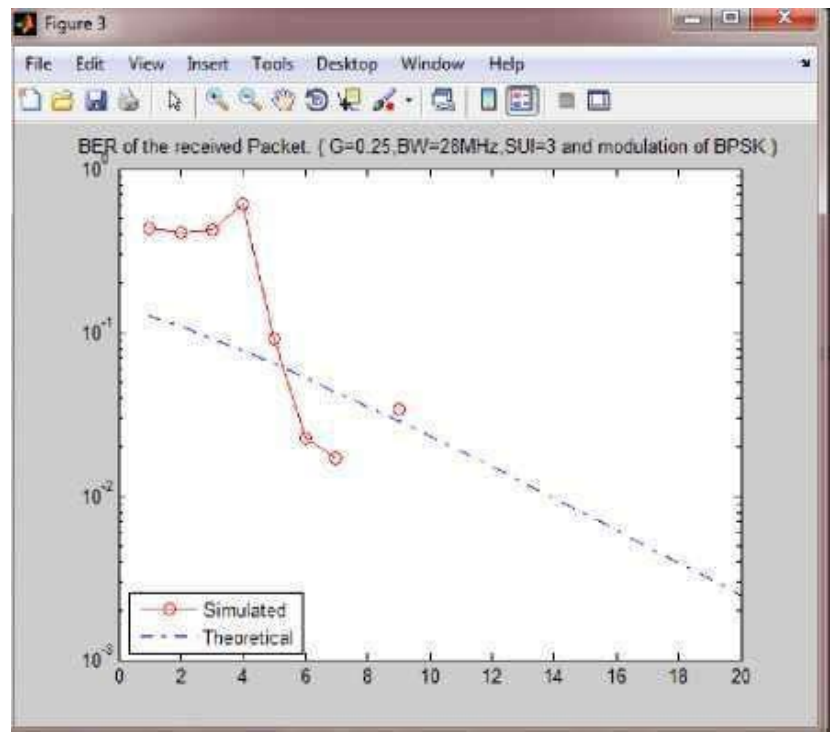

Figure 3: Handover Performance for BPSK channel

$\mathrm{X}$-axis denotes the time lapsed during transmission. Yaxis denotes the BER (Bit Error Rate). Red line -indicates
Hard Handover or Simulated result. Blue line-indicates Soft Handover or Theoretical result.

In fig.3, for BPSK, poor performance is shown as compare

In QPSK very poor performance is shown in figure 4 as data get lost after some time interval \& BER increased as time passes.

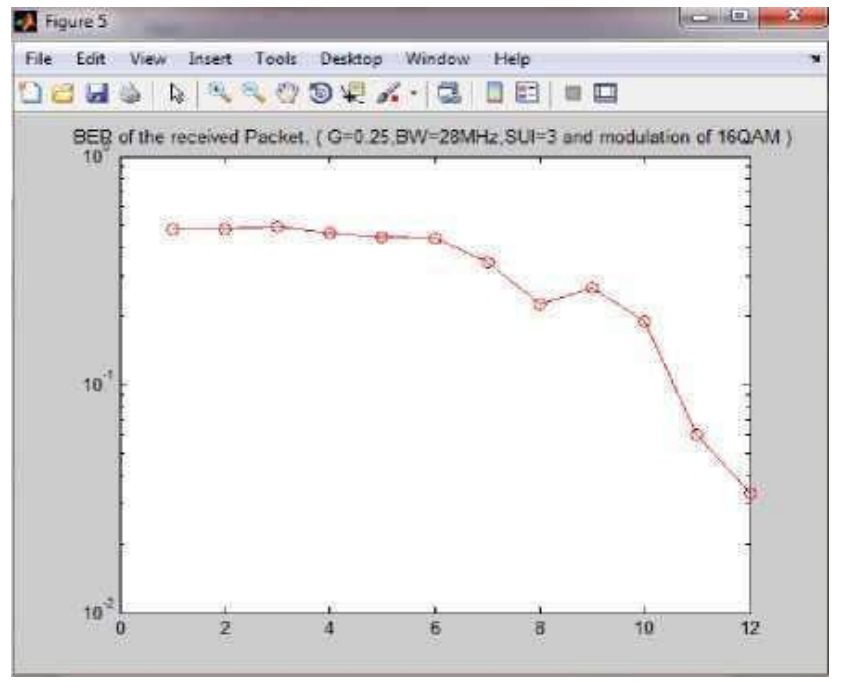

Figure 5: Handover Performance for 16

\section{QAM Channel}

In above simulated figure 5, only hard handover is shown because 16 QAM works at 128 bit in case of hard handover \& thus we got the result. For soft handover, 16 QAM requires to work at 64 bit that's why we doesn't get result for soft handover. Better performance is shown by 16 QAM as compare to other modulation technique like BPSK \& QPSK.

\section{Conclusion}

We simulated the more realistic handover in the mobile WiMAX using MATLAB with WiMAX and mobility modules. The goal of this simulation is to find out the relationship between the handover latency and the velocity of mobile station. It can be seen that the current handover mechanics used in the MATLAB meets the requirement of seamless handover in mobile WiMAX when the mobile station travel at the speed of $20 \mathrm{~m} / \mathrm{s}$. Although, using linkgoing down mechanism will dramatically reduce the handover latency, it is still a challenge to achieve the full mobility: up to $120 \mathrm{~km} / \mathrm{h}$, handover latency of less than 50 ms with an associated packet loss that is less than 1 percent.

\section{References}

[1] Parviz Yegani, "WiMAX Overview," IETF-64 Cisco Systems, November 2005.

[2] IEEE P802.16e/D12, "Air Interface for Fixed and Mobile Broadband Wireless Access Systems: Amendment for Physical and Medium Access Control 


\section{International Journal of Science and Research (IJSR) \\ ISSN (Online): 2319-7064}

Index Copernicus Value (2013): 6.14 | Impact Factor (2015): 6.391

Layers for Combined Fixed and Mobile Operation in Licensed Bands", October 2005.

[3] WiMAX Forum, "Mobile WiMAX - Part I: A technical overview and performance evaluation," August, 2006.

[4] Michael Carlberg Lax and Annelie Dammander WiMAX - A Study of Mobility and a MAC-layer Implementation in GloMoSim.2005

[5] Koodli, R., "Fast Handovers for Mobile IPv6", RFC 4068, 2005.

[6] Zdenek BECVAR, Pavel MACH, Robert BESTAK, Initialization of Handover Procedure in WiMAX Networks.2007

Volume 5 Issue 6, June 2016 www.ijsr.net 